\title{
ADOLESCENCIA Y CÁNCER
}

\section{ADOLESCENCE \& CANCER}

\author{
Ascensión Bellver Pérez ${ }^{1}$ y Helade Verdet Martínez ${ }^{2}$ \\ USSR Hospital Universitario Dr. Peset de Valencia. Universitat de València. \\ 2 Departamento Salud La Fe de Valencia.
}

Resumen

La adolescencia es un período que abarca los de los 15 a 19 años de edad, es una etapa de transición en el desarrollo desde la infancia hasta la vida adulta. La apariencia de los jóvenes cambia como resultado de los acontecimientos hormonales de la pubertad. En esta etapa, incorporan una nueva apariencia y hay una búsqueda de su sentido de identidad. El cáncer en la adolescencia es la principal causa de mortalidad no accidental. Dado que la adolescencia es un período de cambios físicos y emocionales significativos, un diagnóstico de cáncer durante esta etapa del desarrollo tiene un impacto importante en su desarrollo psicológico y físico.

El objetivo es realizar una revisión bibliográfica de los 80 artículos más relevantes publicados durante los últimos años en la literatura asociados con el adolescente con cáncer. La revisión bibliográfica ha sido realizada en las bases computerizadas PubMed, PsycINFOs.

En esta revisión se describe los problemas psicosociales que afrontan los adolescentes con cáncer: problemas de desarrollo, respuestas iniciales al diagnóstico, el ajuste psicológico, la calidad de vida y las secuelas adversas del cáncer, los problemas de imagen corporal, la sexualidad y el cumplimiento y adherencia del tratamiento.

Conclusiones: La falta de recursos personales o experiencias de vida que podrían capacitarlos para afrontar y dar sentido a los muchos problemas potenciales que pueden acompañar

\section{Abstract}

Adolescence covers from the $\mathbf{1 5}$ to the $\mathbf{1 9}$ years of age, is a period of transition in the development from childhood to adult life. The appearance of youth changes as a result of the hormonal events of puberty. At this stage, they incorporate a new look and there is a search for their sense of identity. Adolescent cancer is the leading cause of non-accidental mortality. Adolescence is a significant period of physical and emotional changes and the diagnosis of cancer during this time has an important impact on their psychological and physical development.

The aim is to carry out a literature review of 80 most relevant articles published during the last years in the literature associated with adolescents with cancer. The literature review has been made in computerised databases PubMed, PsycINFOs.

This review describes the psychosocial issues faced by teens with cancer: problems of development, initial responses to the diagnosis, psychological adjustment, quality of life and adverse cancer sequelae, problems with body image, sexuality and the compliance and treatment adherence.

Conclusions: The lack of personal resources or life experiences that might allow them to cope with and make sense of the many potential problems that are inherent to cancer, and sometimes there are not in a period of change and personal development. The article aims to pay attention on the need of making

Correspondencia:

Ascensión Bellver Pérez

USSR. Hospital Universitario Dr. Peset de Valencia. C/Juan de Garay N² 21. 46017 VALENCIA.

E-mail: bellver_asc@gva.es 
el cáncer, es en ocasiones inexistente en un periodo de cambio y desarrollo personal. El artículo pretende llamar la atención sobre la necesidad de creación de unidades especializadas en el tratamiento para adolescentes y la necesidad de que dichas unidades sean multidisciplinares, formadas por profesionales especialistas en este complejo periodo del desarroIlo e incluyan profesionales de la Salud Mental.

Palabras clave: Cáncer, adolescente, adaptación psicológica, intervención psicológica. specialised units in treatment of adolescents with multidisciplinary specialists, which ones were specialized in this complex period of development and that include mental health professionals.

Keywords: Cancer, adolescent, psychological adjustment, psychological intervention.

\section{INTRODUCCIÓN}

La adolescencia es un periodo de transición desde la niñez a la edad adulta que se caracteriza por grandes cambios psicológicos, biológicos y sociales, que suponen desequilibrios y ajustes. Los adolescentes tienen que desarrollarse física, psicológica, sexual y socialmente.

Petersen, Kennedy y Sullivan (1991) describieron el principio de la adolescencia como una época de "transiciones" del desarrollo, incluyendo el cambio de la educación primaria a la secundaria, los cambios en las expectativas de los compañeros, una vida con mayor estrés, y cambios en las relaciones y roles dentro de la familia y en otros contextos. Estos autores también se refirieron a los cambios internos a nivel cognitivo, así como a las habilidades y las transformaciones físicas de la pubertad $^{(1)}$.

Estos cambios incluyen cada vez más independencia de los adultos, como padres y maestros, y estar cada vez más sujeto a las evaluaciones sociales de los compañeros $^{(2)}$. La independencia se centra en la apariencia y la autoimagen, el desarrollo de las relaciones íntimas, el ajuste al crecimiento de la pubertad, así como el establecimiento de metas para el futuro ${ }^{(3)}$.

Desde la perspectiva psicológica, se describe como un periodo de vida donde se van alcanzando hitos tales como el es- tablecimiento de la personalidad y la identidad del rol sexual, así como el desarrollo de la moral y la independencia emocional, además las relaciones entre iguales se convierten en algo cada vez más importante $^{(4)}$. El intento de los adolescentes de lograr una postura más independiente y dejar atrás la intensa dependencia de los padres, trae consigo una especial vulnerabilidad en un adolescente enfermo, que se esfuerza por obtener la independencia de sus padres y ahora depende de maneras nuevas y diferentes ${ }^{(5)}$.

La adolescencia es una etapa de transición entre la niñez y la edad adulta. La Organización Mundial de la Salud (OMS) define los adolescentes como individuos entre los 10 y 20 años de edad, mientras que en los Estados Unidos la adolescencia se definiría entre la edad de 13 a 19 años. A los efectos de estudios de epidemilogía y de la investigación del cáncer, el National Cancer Institute $(\mathrm{NCl})$ considera que el periodo de adolescencia esta asignado el rango de edad de 15 a 19 años. A si mismo, Barr declaró que, en el contexto del cáncer, la adolescencia abarca el período entre 15 y 19 años ${ }^{(6)}$. Los datos europeos y estadounidenses muestran que la supervivencia global a los 5 años en adolescentes con cáncer es de aproximadamente 73$78 \%$. Los adolescentes tienen una supervivencia más baja que los niños debido a la leucemia linfoide aguda, linfoma maligno 
no Hodgkin, tumor de Ewing, osteosarcoma y al sarcoma de partes blandas ${ }^{(7)}$.

El adolescente con cáncer tienen que tratar con dos fuentes de estrés: tener un diagnóstico que pone en peligro la vida y su tratamiento intensivo asociado, además del desafío de la transición del desarrollo desde la niñez a la edad adulta ${ }^{(8)}$. Además, la adolescencia es generalmente una fase del desarrollo que comprende una mayor vulnerabilidad al estrés emocional que podría verse seriamente agravada por la experiencia del cáncer. Esto puede ser abrumador para muchos adolescentes y afectar a su capacidad para hacer frente a las tareas normativas del desarrollo ${ }^{(9)}$.

El cáncer en los adolescentes plantea una serie de retos únicos tanto para el paciente como para sus familiares. El diagnóstico de cáncer durante este tiempo tiene un gran impacto en su bienestar psicológico y el desarrollo físico. El tiempo fuera de la escuela y de otras actividades puede costar amistades y crear sentimientos de aislamiento en un momento en el que sentirse conectado a sus compañeros es de suma importancia para el sentido de identidad del adolescente ${ }^{(6)}$.

El diagnóstico de cáncer, el tratamiento invasivo asociado y los subsiguientes efectos secundarios extensos tales como la pérdida de cabello, pérdida de peso, diarrea, náuseas, vómitos, cambios en la piel, fatiga, dolor o insomnio están asociados con una fuerte tensión y carga psicológica ${ }^{(9,10)}$. Estos recordatorios visibles del cáncer tienen el potencial de dañar seriamente no sólo la salud de la persona joven, sino también su sentido de desarrollo de la propia identidad y la imagen corporal ${ }^{(10)}$.

El período inmediatamente posterior a un diagnóstico de cáncer durante la adolescencia es muy estresante ${ }^{(11)}$. Sin embargo, a partir de los18 meses después del diagnóstico, los adolescentes informan de una mejor función psicosocial en comparación con su grupo de referencia ${ }^{(11)}$. A pe- sar de estos hallazgos, sin embargo, hay un sub-grupo de adolescentes con cáncer que informan de un pobre ajuste ${ }^{(12)}$.

Esto es potencialmente problemático, ya que el grado en que estos adolescentes con cáncer tienen éxito para hacer frente a la enfermedad y a la angustia relacionada con el tratamiento puede influir en su capacidad para dominar las tareas apropiadas para su edad y de ese modo lograr metas sociales a largo plazo ${ }^{(13)}$.

Los adolescentes con cáncer tienen que hacer frente a los efectos adversos del tratamiento, los cambios en las relaciones familiares, el aislamiento social, la ruptura en la educación, las disfunciones sexuales y las cuestiones laborales. Por tanto, psiquiatras, psicólogos y trabajadores sociales deben participar activamente en su apoyo $^{(14)}$.

Aunque muchos de los adolescentes parecen estar resilientes, una parte de los pacientes en este grupo de edad experimentan dificultades clínicamente relevantes en la adaptación a su diagnóstico de cáncer $^{(13)}$. Por lo tanto, son necesarios los servicios especializados de intervención y la elaboración de programas multidisciplinares. Esos programas deben abordar los problemas comunes, como la escuela, el trabajo y la integración escolar y social, así como hacer frente a las deficiencias físicas, la imagen corporal y la asertividad debido a los efectos tardíos como la amputación u otras discapacidades físicas ${ }^{(9)}$. La prestación de apoyo psicológico y social es hoy en día una parte integral del tratamiento del cáncer. La Sociedad Internacional de Oncología Pediátrica (SIOP) publicó una serie de directrices para los problemas psicosociales en el cáncer pediátrico ${ }^{(15)}$.

Los adolescentes tienden a tener un fuerte sentido de independencia y rebeldía, y pueden ser reacios a confiar en el sistema médico. Pueden retrasar el hablar acerca de sus síntomas a los padres y médicos, e incluso, cuando ven al médico, 
pueden dar información errónea ${ }^{(16)}$. El intervalo entre el comienzo de los primeros síntomas y el diagnóstico de cáncer es más largo en los adolescentes que en los niños ${ }^{(17,18)}$.

Las necesidades de los adolescentes con cáncer sólo pueden abordarse mediante personas específicamente entrenadas, con el apoyo de unidades de hospitalización especializadas. Asimismo, es necesaria la investigación dedicada a programas específicos para este período, y que no sean parte de la atención para niños o adultos $^{(16)}$. Hay pruebas de que la centralización del tratamiento para adolescentes en unidades especializadas mejora las tasas de curación y los resultados del tratamiento para estos pacientes ${ }^{(19)}$. Los adolescentes con cáncer que logran un cierto sentido de control sobre su tratamiento y la responsabilidad por sí mismos son más propensos a cumplir con el tratamiento ${ }^{(5)}$.

El objetivo del presente estudio es realizar una revisión de los artículos más relevantes publicados en los últimos años que hacen referencia a los aspectos psicosociales del cáncer en adolescentes y resaltar la necesidad de una intervención psicosocial en este grupo de edad, teniendo en cuenta tanto el impacto del cáncer como las necesidades específicas de esta etapa del desarrollo. La revisión bibliográfica ha sido realizada en las bases computerizadas PubMed y PsycINFO empleando las palabras clave adolescencia, cáncer, adaptación psicológica e intervención psicológica, seleccionando los artículos desde el año 1980 al año 2012.

\section{Alteraciones emocionales y estrategias de afrontamiento}

La adolescencia es, generalmente, una fase de desarrollo que comprende una mayor vulnerabilidad al estrés emocional que podría verse seriamente agravada por la experiencia del cáncer. Esto puede ser abrumador para muchos adolescentes y afectar a su capacidad para hacer frente a las tareas normativas del desarrollo. Dadas las dificultades particulares que tienen que afrontar los adolescentes con cáncer, es importante hacer frente a las necesidades emocionales de estos pacientes ${ }^{(20)}$.

El diagnóstico de cáncer, que supone un peligro para la vida, el tratamiento invasivo asociado y los subsiguientes efectos secundarios, hacen que los pacientes tengan que enfrentar su posible muerte y la necesidad de desarrollar nuevas estrategias de afrontamiento para hacer frente a todos los nuevos retos de la situación. Además, las numerosas visitas al médico y las hospitalizaciones pueden trastornar la rutina cotidiana del adolescente y su familia y afectar a sus roles sociales ${ }^{(20)}$.

El cáncer es una experiencia traumática que despierta sentimientos de ira, ansiedad, miedo y tristeza. Para el adolescente con cáncer, se pierden oportunidades, no sólo en la vida diaria, sino también a través de pérdidas de eventos sociales como salidas con los amigos. Los pacientes adolescentes pueden entender las implicaciones del diagnóstico de cáncer, pero carecen de los recursos personales o experiencias de vida que podría capacitarlos para afrontar y dar sentido a los muchos problemas potenciales que pueden acompañar el cáncer ${ }^{(21)}$. Una enfermedad crónica, como el cáncer, trae consigo importantes cambios en el estilo de vida, que demandan nuevas estrategias de afrontamiento en estos adolescentes.

Wu et al. compararon adolescentes con cáncer en tratamiento con otro grupo sin tratamiento y con un grupo control sano. Los que estaban en tratamiento informaron de una calidad de vida más pobre en general. De los que estaban en tratamiento, las mujeres también informaron de un funcionamiento psicológico y cognitivo y una visión de la vida más pobre que las mujeres sanas ${ }^{(22)}$. 
Koocher et al. investigaron 115 adolescentes con cáncer que habían estado en remisión durante por lo menos un año. Se encontró un número significativo de supervivientes que experimentaban dificultades residuales en el funcionamiento psicológico, el 59\% mostró síntomas psiquiátricos leves y el $12 \%$ estaban notablemente o gravemente afectados psicológicamente. El efecto secundario más común fueron la baja autoestima, la depresión y la ansiedad. Además los individuos que eran adolescentes en el momento del diagnóstico eran propensos a tener problemas en el ajuste psicosocial, la socialización, las habilidades de autoayuda y el funcionamiento intelectual ${ }^{(23)}$.

Hay diversos estudios en niños y adolescentes que estiman que entre un 17$30 \%$ de los adolescentes con cáncer describen angustia psicológica significativa, incluyendo síntomas de ansiedad y depresión, y que estas cifras son similares entre población pediátrica y adolescente con cáncer ${ }^{(24-26)}$. Esto se confirma en estudios más recientes realizados a largo plazo con supervivientes adolescentes mostrando una prevalencia tan alta como el $24,3 \%$ en trastornos clínicos tales como la ansiedad, depresión o trastorno por estrés postraumático (TEP), con distrés subclínico también prevalerte en un $22,4 \%{ }^{(27)}$. Más que los supervivientes de cáncer infantil, personas diagnosticadas con cáncer durante la adolescencia o edad adulta joven tienen la capacidad cognitiva de entender la gravedad de su enfermedad y demuestran con frecuencia angustia y ansiedad persistente sobre la muerte, la recurrencia del cáncer o los efectos tardíos. Alteraciones en el aspecto físico, cambios de peso, pérdida de cabello, amputaciones, colocación de catéteres para facilitar la administración del tratamiento, cicatrices quirúrgicas y alteraciones en la coloración de la piel y la textura hacen que los adolescentes se sientan diferentes a sus compañeros ${ }^{(28)}$.
Una revisión reciente de pacientes pediátricos con cáncer inscritos en ensayos clínicos en el (NIH) mostró que un 14\% tenían prescrito al menos un psicofármaco (ISRS) para tratar los síntomas de depresión y ansiedad. Muchos de los medicamentos utilizados en el tratamiento de los síntomas psiquiátricos pueden tener beneficios complementarios en los síntomas asociados con el cáncer y los efectos secundarios relacionados para su tratamiento. Por ejemplo, las benzodiazepinas son con frecuencia utilizadas para tratar la ansiedad y náuseas debido a los agentes quimioterapéuticos. Los medicamentos estimulantes pueden usarse para tratar la fatiga $y$ el letargo en pacientes adolescentes con cáncer ${ }^{(29-30)}$.

Bleyer mostró que los efectos secundarios del tratamiento del cáncer son más graves y difíciles de sobrellevar durante la etapa de la adolescencia y que, durante el tratamiento activo, la calidad de vida es peor $^{(31)}$. Los adolescentes han demostrado de forma consistente mayores tasas de incumplimiento y baja adherencia respecto tanto a los niños como a los adultos en el tratamiento del cáncer. El cumplimiento y adherencia del adolescente con los tratamientos oncológicos tienen el potencial de convertirse en un campo de batalla donde la etapa del desarrollo entra en conflicto con las necesidades del tratamiento médico $^{(5)}$.

Von Essen et al. encontraron que el impacto del diagnóstico de cáncer depende de la etapa de tratamiento: mientras pacientes pediátricos con cáncer en tratamiento no difirieron de controles sanos en cuanto a sus puntuaciones en ansiedad y depresión, los pacientes sin tratamiento mostraron puntuaciones más altas en ambas variables, así como un menor bienestar psicológico y una menor autoestima en comparación con la población control sana ${ }^{(32)}$.

Sin embargo, otros estudios han obtenido resultados contradictorios. Kaplan et 
al. observaron que la mayoría de los pacientes adolescentes con cáncer y los sobrevivientes no están más angustiados, en términos de niveles de ansiedad y depresión, y no tienen más deterioro psicosocial que la población general ${ }^{(33)}$. Allen et al. no encontraron diferencias significativas en las puntuaciones de depresión y ansiedad en el grupo de cáncer en relación con un grupo control saludable emparejado por edad, aunque algunos participantes de ambos grupos tenían puntuaciones elevadas en ansiedad y depresión ${ }^{(25)}$.

Se han identificado factores de riesgo en los adolescentes que favorecerán la aparición de problemas para hacer frente a un diagnóstico de cáncer: las dificultades de afrontamiento de los padres, tener problemas sociales o emocionales antes del diagnóstico y un estilo atribucional depresivo $^{(34)}$. La alta angustia en los padres ha sido fuertemente correlacionada con la desesperanza en sus hijos ${ }^{(35)}$. Los resultados positivos del tratamiento, la participación en la vida familiar, las aficiones y los estudios promueven una buena adaptación. Muchas personas con cáncer también utilizar estrategias que no les ayudan a hacer frente a la enfermedad, sino que les ayudan a tolerar la situación (impotencia y creencia en el destino). Estas estrategias suelen provocar ansiedad y depresión y problemas en el manejo de las nuevas situaciones estresantes ${ }^{(36)}$.

El tipo de cáncer parece jugar un papel importante también en determinar el impacto psicológico. Los cánceres del sistema nervioso central, en particular, están asociados con mayores niveles de distrés psicológico. Esto puede, en parte, ser debido a la mayor carga de déficits neurocognitivos y el mayor grado de dificultades sociales experimentados por los pacientes con tumores cerebrales adolescentes durante y después del tratamiento ${ }^{(37)}$. Los tumores óseos y osteosarcomas son también muy comunes con un pico de incidencia a la edad de 15 años. Desafortunadamente, muchos de los adolescentes afectados tienen que lidiar con graves déficits funcionales debido a la reconstrucción de la extremidad o la amputación, además de los efectos de la quimioterapia (por ejemplo los déficits en el desarrollo) ${ }^{(6)}$. Cabría esperar que el pronóstico sea un importante predictor de ajuste, aunque parecen haber pocos datos para apoyar esta hipótesis. Sin embargo, una mayor incertidumbre en el resultado del tratamiento, como en el uso de procedimientos experimentales, se ha correlacionado con una mayor distrés psicológico ${ }^{(26)}$.

Respecto a las estrategias de afrontamiento empleadas, Kyungäs et al. encontraron que las principales estrategias de afrontamiento utilizadas por los adolescentes con cáncer fueron el apoyo social, la creencia en la recuperación y volver a la vida normal tan pronto como sea posible. Por otra parte, una actitud de vida positiva, la creencia en los propios recursos, la creencia en dios, y la voluntad de luchar contra la enfermedad fueron los recursos para hacer frente al cáncer(38). En el estudio realizado por Engvall et al., en respuesta a las preguntas cerradas, la mayoría de los adolescentes informaron de que habían usado estrategias de afrontamiento basadas en la emoción (aceptación y minimización), mientras que, en respuesta a las preguntas abiertas, informaron de que habían empleado más estrategias basadas en el significado (pensamiento positivo) y centradas en el problema (resolución de problemas). La mayoría informaron de haber usado la minimización y la búsqueda de ayuda en respuesta a las preguntas cerradas, pero muy pocos adolescentes mencionaron el uso de estas estrategias en respuesta a las preguntas abiertas ${ }^{(39)}$. La esperanza juega un papel importante para hacer frente al diagnóstico y el tratamiento del cáncer. El nivel de optimismo ha correlacionado con un sentido positivo de 
bienestar y compromiso con el tratamiento y también mejora la supervivencia y la autoestima $^{(40)}$.

Un aspecto importante de la vida de un adolescente es formar parte de un grupo de pares. Las relaciones estrechas entre pares son una fuente importante de apoyo para los adolescentes con enfermedades crónicas. Mientras que los padres continúan desempeñando el papel de los cuidadores primarios, los amigos y los compañeros proporcionar apoyo emocional mediante la aceptación de su amigo enfermo y de sus limitaciones físicas ${ }^{(39)}$. Los adolescentes con cáncer y sus familias necesitan apoyo social para hacer frente a su enfermedad y los cambios que ha causado. El apoyo social que se recibe de los padres, amigos y profesionales de la salud es el recurso más importante para los adolescentes con cáncer ${ }^{(36)}$.

El personal de oncología juega un papel importante en el apoyo de los pacientes y sus familias. El personal de salud ocupa un lugar privilegiado para reconocer los desafíos psicosociales únicos que enfrentan los adolescentes. Sin embargo, los problemas de carácter psicosocial con frecuencia son sobrestimados por el personal. Tanto los médicos como las enfermeras suelen ser bastante precisos en estimar la angustia física en adolescentes con cáncer, sin embargo, ambos sobreestiman los niveles de ansiedad y depresión. Así, la identificación y la adopción de medidas en relación al malestar psicológico deben basarse en la comunicación directa entre los adolescentes y el personal ${ }^{(41)}$.

\section{Autoestima, imagen corporal, relaciones sexuales y fertilidad}

La adolescencia y el inicio de la edad adulta es el momento en que se produce la experimentación personal, el desarrollo de nuevas relaciones, la exploración de la conciencia sexual y la iniciación de la actividad sexual, considerándose tareas esenciales en esta etapa del desarrollo. La autoestima y la salud sexual tienen un impacto significativo en la formación de la identidad del adolescente, especialmente en aquellos pacientes jóvenes que tienen que hacer frente a un diagnóstico de cáncer. El conocimiento de la salud sexual, las relaciones interpersonales y los problemas de imagen corporal son factores que tienen un impacto en el desarrollo de la autoestima durante la transición a la edad adulta ${ }^{(42)}$. Durante el desarrollo de este período, un adolescente con cáncer puede experimentar ciertos desafíos en el establecimiento de una identidad sexual positiva ${ }^{(28)}$.

Obstáculos al acceso del conocimiento de una adecuada salud sexual, dificultades en las relaciones interpersonales ${ }^{(38,43)}$, y las preocupaciones de la imagen corporal(44) pueden ser dificultades que los adolescentes y adultos jóvenes con cáncer tienen que afrontar y comprometen el desarrollo de una adecuada autoestima(45). La merma de las relaciones sociales con el grupo de iguales debido a las continuas visitas clínicas, tratamientos o infecciones que requieren continuos ingresos hospitalarios y tiempo perdido de asistencia al aula, se añaden a las dificultades en el desarrollo de un sentido de sí mismo y los sentimientos de agrado de uno mismo ${ }^{(42)}$. La carga adicional de los efectos secundarios del tratamiento de cáncer tal como la alopecia o la pérdida de peso, cicatrices, efectos cutáneos, trastornos del crecimiento o infertilidad pueden influir en la imagen corporal, la autoestima y la identidad sexual del adolescente ${ }^{(46)}$.

La formación de la identidad sexual y la capacidad reproductiva son claves en las tareas del desarrollo en la adolescencia. El impacto del diagnóstico y tratamiento del cáncer, tanto en la fertilidad, como en la identidad psicosexual, puede ser potencialmente devastadores, sobre todo si no son detectados y atendidos en esta etapa ${ }^{(47)}$. 
Se ha visto que los supervivientes de cáncer infantil experimentan retrasos en las citas e iniciar contactos sociales ${ }^{(48,49)}$, en el matrimonio ${ }^{(50,51)}$ y en la primera vez que tienen relaciones sexuales ${ }^{(52,53)}$. Suelen ser menos propensos a ser sexualmente activos y tal vez menos satisfechos con las relaciones interpersonales y la vida sexual ${ }^{(54)}$.

Normalmente, cuando un niño madura, los padres ajustan sus prácticas de supervisión para permitir más libertad e independencia en la toma de decisiones por parte del adolescente ${ }^{(55)}$. Sin embargo, los padres de adolescentes con cáncer tienden a ser más sobreprotectores con sus hijos. Con la experiencia de la enfermedad de su hijo, los padres podrán ver a su hijo como particularmente vulnerable para las relaciones interpersonales o el inicio de las relaciones sexuales ${ }^{(56)}$. Del mismo modo, el adolescente puede experimentar esta sobreprotección no sólo por parte de los miembros de la familia incluso también por parte de sus compañeros de clase y profesores. Esta sobreprotección y el mensaje de vulnerabilidad pueden desalentar al adolescente en la realización de esfuerzos hacia la individuación y el desarrollo de su propia identidad sexual ${ }^{(56)}$.

Schover resume los impactos fisiológicos de los tratamientos de cáncer en la sexualidad en que los tratamientos contra el cáncer pueden dañar uno o más sistemas fisiológicos necesarios para una respuesta sexual sana, incluyendo el sistema hormonal, elementos vasculares, neurológicos y psicológicos de la función sexual. Como resultado de estos factores, la disfunción sexual puede ser caracterizada por trastornos en el deseo sexual y/o en los cambios psicofisiológicos asociados con la respuesta sexual humana(57). Mientras que las mujeres supervivientes pueden describir más síntomas sexuales que los hombres supervivientes, los hombres pueden experimentar más malestar asociado con las dificultades sexuales ${ }^{(46)}$.
Cuestiones como la preservación de la fertilidad pueden tener poco peso o interés en un joven adolescente ${ }^{(58)}$, pero puede ser de interés primordial para la misma persona en la vida adulta ${ }^{(46)}$. Un joven de 13 años de edad que no haya empezado a masturbarse puede verse enfrentado a tomar una decisión de tener su esperma congelado al mismo tiempo, y en medio, de tener que hacer frente a un diagnóstico y tratamiento del cáncer. Durante la etapa de la adolescencia, no es raro que los jóvenes presten poca atención a su potencial fertilidad $^{(43)}$. Es importante plantear este problema con conciencia de que el joven se beneficiará de discutir a fondo sobre el grado real de riesgo para su potencial reproductivo y sobre las opciones disponibles para la preservación de la fertilidad. La evaluación objetiva y la comunicación honesta son fundamentales en la orientación de una persona joven y su familia en la toma de decisiones en torno a la fertilidad. Se debe hacer un esfuerzo para evitar la impresión de que las discusiones y decisiones son apresuradas o urgentes, y el asesoramiento debería efectuarse de forma accesible a los jóvenes para el apoyo en la toma de decisiones.

Las tecnologías que están disponibles para la preservación de la fertilidad para los adolescentes con cáncer en la mayoría de los casos deben llevarse a cabo antes del tratamiento con quimioterapia ${ }^{(59)}$. La opción establecida (no experimental) para los hombres es la criopreservación de espermatozoides, y para las mujeres las opciones establecidas son la transposición ovárica y la criopreservación de embriones. Hay opciones experimentales disponibles tanto para hombres como para mujeres como son la criopreservación de ovocitos, testicular, y la criopreservación de tejido ovárico ${ }^{(59)}$. En algunos casos, el médico puede decidir que para un paciente adolescente no es posible retrasar el tratamiento del cáncer y de no poder 
establecer una opción de preservación de la fertilidad. Sin embargo, la necesidad de considerar la preservación de la fertilidad depende en ocasiones de varios factores como la capacidad física y de desarrollo del paciente, el pronóstico, circunstancias económicas y los valores religiosos, culturales y éticos ${ }^{(60)}$. Se debe prestar especial atención a los valores y deberes de las partes interesadas y a los potenciales conflictos que pueda surgir.

Aunque es logísticamente más sencillo para los varones sexualmente maduros, la preservación de la fertilidad no está exenta de problemas, y el banco de esperma no debe ser minimizado por su capacidad de ser particularmente estresantes para él, estrés que se suma a un ya muy cargado contexto de enfermedad en que les toca vivir $^{(61)}$. Pero los desafíos para la conservación de la fertilidad en las mujeres jóvenes es más complejo y difícil con algunas técnicas probadas disponibles en la actualidad $^{(62)}$. Las mujeres jóvenes pueden necesitar apoyo para llegar a un acuerdo sobre los riesgos para su fertilidad. La toma de decisiones sobre preservación de la fertilidad puede ser muy difícil para los jóvenes que aún no se han considerado su futuro como padres, sobre todo en una situación en la que han de enfrentarse a su mortalidad. Por lo tanto, es importante que estos temas se traten y haya información a su disposición, en particular en su transición hacia la supervivencia. Consejo, apoyo e información acerca de la fertilidad y la evaluación de la reproducción asistida debe estar disponible para afrontar la realidad de sus decisiones y la forma en que influirá en sus vidas adultas ${ }^{(47)}$.

\section{Intervención psicológica en adolescentes con cáncer}

Las mejoras en los tratamientos médicos del cáncer han conllevado que surja una creciente población de supervivientes en la edad del adolescente y joven adulto que han superado la enfermedad ${ }^{(7)}$. Se ha visto que los supervivientes adolescentes de cáncer tienen una peor calidad de vida que los supervivientes de cáncer infantil ${ }^{(63)}$, y que su grupo de iguales sanos ${ }^{(64)}$. Los supervivientes adolescentes han mostrando a largo plazo una prevalencia alrededor del 24,3\% en trastornos clínicos tales como la ansiedad, depresión o trastorno por estrés postraumático $(\mathrm{TEP})^{(27)}$. Sin intervención, estos problemas de salud mental representan una amenaza significativa para el ajuste psicológico de los supervivientes a más largo plazo. Programas de apoyo psicosociales apropiados y basados en la evidencia están fuertemente indicados para los supervivientes adolescentes enfermos de cáncer ${ }^{(65)}$.

Las intervenciones psicológicas adyuvantes están indicadas para reducir la angustia y malestar psicológico relacionado con el cáncer en la adolescencia, son por lo tanto una parte fundamental de tratamiento multidisciplinario. Desde la PsicoOncología se tiene que considerar los aspectos del desarrollo específicos de la adolescencia, esta revisión resume los estudios empíricos que han valorado la eficacia y la efectividad de las intervenciones psicosociales para los pacientes adolescentes con cáncer.

En la variedad de intervenciones con adolescentes con cáncer, et al. se plantean como objetivo la mejora del área psicosexual ${ }^{(66)}$. El objetivo del componente educativo era facilitar información sobre el impacto de la terapia del cáncer sobre el desarrollo sexual y la fertilidad, ayudar a los participantes a explorar el impacto de sus tratamientos del cáncer en las relaciones entre iguales y la identidad sexual en adolescentes con enfermedad hematológica y tumores sólidos. Se realiza mediante intervenciones individuales de asesoramiento en 2 sesiones, con grupo intervención y grupo control en lista de espera. Los resul- 
tados demuestran un aumento significativo de los conocimientos sobre el efecto del cáncer sobre la sexualidad y fertilidad, la mejora de la imagen corporal, menos ansiedad y disminución en el nivel general de malestar psicológico en la evaluación posterior a la intervención en el grupo experimental ${ }^{(66)}$.

El objetivo del estudio piloto de Baider y Kaplan De-Nour con adolescentes con cáncer estaba encaminado a trabajar las estrategias de afrontamiento y preparar a los participantes para facilitar el apoyo mutuo. Se facilitaba la experiencia de compartir y discutir preocupaciones motivadas por su diagnóstico y su tratamiento de cáncer, así como su patrón de afrontamiento individual en sesiones de grupo de orientación psicodinámica. Los resultados de Baider y Kaplan De-Nour parecían no haber descubierto cambios significativos en el grupo de intervención a lo largo del tiempo. Sin embargo, cuando se comparaba a los adolescentes que estaban en tratamiento activo del cáncer y aquellos que no, considerados por separado, aumentaban las medidas de distrés psicológico para pacientes en tratamiento activo y disminuía en los pacientes que no estaban en tratamiento ${ }^{(67)}$.

Hay una extensa evidencia de estudios que apoyan la eficacia de la Terapia Cognitivo Conductual en el tratamiento de ansiedad y depresión en adolescentes afectados de cáncer, así como en la prevención de dichos trastornos ${ }^{(68,69)}$. Un meta-análisis de 130 estudios mostró que este tipo de programas normalmente consigue efectos al menos moderados ${ }^{(70)}$. No hay estudios que hayan examinado la eficacia de la terapia grupal cognitivo-conductual en una población de adolescentes con cáncer. Datos experimentales sugieren, sin embargo, que adolescentes con cáncer responden positivamente a las habilidades y técnicas cognitivo-conductuales y que incorporan estas habilidades en su vida cotidiana. En consonancia con esto, una revisión reciente de las intervenciones con adolescentes con enfermedades crónicas, (incluyendo cáncer) basadas en competencias altamente eficaces en el tratamiento de problemas emocionales o conductuales, muestran que producen un efecto de mediano a gran$\mathrm{de}^{(71)}$. Estos datos recomiendan la terapia cognitivo-conductual en grupo como un tratamiento prometedor, como forma de intervención psicológica en adolescentes que conviven con cáncer ${ }^{(71)}$. También se resalta la importancia de la modalidad de terapia: entre grupo de apoyo de iguales basada en terapia dirigida a adolescentes y adultos jóvenes supervivientes del cán$\operatorname{cer}^{(72)}$. Los grupos de apoyo de iguales pueden abordar el sentimiento de aislamiento y desarrollar la conexión con su grupo de iguales de la misma edad, facilitando el contacto con pacientes dispersos, mientras están en tratamiento ${ }^{(73)}$. De hecho, son muy receptivos a terapias de apoyo mutuo como una forma de intervención ${ }^{(74)}$. La opción de unidades de apoyo para la discusión y para compartir estrategias de adaptación entre adolescentes, resulta positivo. Es importante evaluar el aislamiento geográfico y demográfico y ante demandas buscar soluciones innovadoras para facilitar la interacción entre iguales, teniendo en cuenta que los adolescentes actuales son usuarios de Internet (entre 1-3 horas de uso diario), y realizan muchas de sus interacciones entre iguales en línea ${ }^{(75)}$, lo que lleva a desarrollar terapias apoyadas en Internet. El uso de Internet en psicoterapia, ha sido aclamado como una nueva forma de superar problemas de equidad en la accesibilidad y el estigma asociado con la búsqueda de ayuda de profesionales de la salud mental por parte de los jóvenes ${ }^{(76)}$. Cada vez hay un mayor desafío en ofrecer programas basados en competencias mediante las nuevas tecnologías y modelos de intervención, que valoran el uso de la terapia cognitivo-conductual gru- 
pal, vía Internet en los jóvenes después del tratamiento contra el cáncer, demostrando mejoría en la calidad de vida ${ }^{(77)}$.

Un novedoso programa de intervención para los adolescentes con cáncer es el programa descrito por Shama y Lucchetta. Este programa ofrece una innovadora intervención terapéutica mediante la conexión entre los adolescentes con cáncer, ayudando a superar el aislamiento social haciéndoles participar en eventos 'normales'. La respuesta registrada hasta el momento es prometedora ${ }^{(78)}$. Por otra parte, Barrera et al. describen dos grupos de intervención y apoyo para jóvenes con cáncer. Para uno de esos programas pilotos, valoran los síntomas de ansiedad y depresión pre y post intervención, así como habilidades sociales; se trata de un programa en desarrollo de que desafortunadamente todavía no se han descrito resultados ${ }^{(79)}$.

Hay una serie de limitaciones en los estudios anteriormente descritos, que necesitan ser consideradas. En primer lugar, cabe señalar que la mayoría de los estudios se basaban en muestras relativamente pequeñas, parcialmente debidas a su carácter de proyecto piloto. Analizando en conjunto, los estudios que valoran las intervenciones psicológicas basadas en evidencias para adolescentes con cáncer en la actualidad son todavía muy escasos. Hay muy pocos estudios identificados que valoren la intervención psicológica para el adolescente con cáncer con medidas de resultado estandarizadas. No hay duda que existe una gran brecha entre las intervenciones que están clínicamente disponibles para los adolescentes con cáncer y las pocas que empíricamente se han investigado ${ }^{(80)}$.

\section{CONCLUSIONES}

Los adolescentes con cáncer no cuen$\tan$ con los recursos de afrontamiento necesarios para hacer frente a una situación que pone en riesgo la vida y que implica un importante cambio en su estilo de vida. El diagnóstico de cáncer supone una nueva carga en un momento de la vida en el que el adolescente trata de ganar autonomía y establecer una identidad independiente

Algunos estudios no encuentran diferencias entre un grupo control y un grupo de adolescentes con cáncer en la presencia de alteraciones emocionales ${ }^{(32,33)}$. Sin embargo, otros autores sí han encontrado estas diferencias ${ }^{(5,22,23,31)}$. Esta disparidad de resultados puede estar indicando que, aunque en general los adolescentes con cáncer no desarrollan alteraciones emocionales, existe un subgrupo que sí las están presentando.

Hemos de tener en cuenta que la adolescencia es una etapa del desarrollo en la que existe una mayor vulnerabilidad al estrés y que los adolescentes con cáncer deben hacer frente a una mayor carga que puede afectar a la consecución de las tareas normativas del desarrollo, se hace necesaria una atención especializada para detectar la presencia de las posibles alteraciones emocionales que puedan presentar.

Se han identificado como factores de riesgo la angustia de los padres y las dificultades de afrontamiento de los mis$\operatorname{mos}^{(25,34)}$. Las estrategias de afrontamiento por parte de los adolescentes que ayudan a prevenir las alteraciones emocionales y que podrían ser trabajadas por los equipos y unidades de oncología serían el apoyo social, la creencia en la recuperación y la vuelta a su vida cotidiana lo antes posible ${ }^{(36)}$.

Tanto el diagnóstico de cáncer como el tratamiento consiguiente son particularmente difíciles para los pacientes adolescentes. En las instituciones tanto pediátricas y como de adultos, a menudo existe una falta de conciencia de los problemas y necesidades psicosociales de los jóvenes con cáncer. Esto significa que los adolescentes están rodeados de pacientes mucho mayores o más jóvenes que ellos mismos, que aumenta su sentido de aislamiento y 
refuerza su percepción de que son diferentes de sus compañeros. Las intervenciones psicológicas orientadas a reducir la angustia y malestar psicológico deberían ser una parte fundamental del tratamiento multidisciplinar. Para ello, sería necesaria la inclusión de los profesionales de salud mental en los equipos, con el objetivo de detectar los problemas y necesidades psicosociales y construir una alianza terapéutica con el adolescente y la familia para apoyarles en el afrontamiento de los problemas que puedan surgir durante todo el proceso.

La atención médica, a diferencia de cualquier otra parte de la sociedad occidental, tiene en gran parte a los adolescentes ignorados como un grupo que requiere especial atención, se encuentra entre las estructuras asistenciales de áreas pediátricas y la de los modelos de adultos. Sin embargo, cada vez se reconoce que los adolescentes dentro de los sistemas de atención de salud requieren servicios específicos por edad, incluyendo la transición de pediatría a servicios para adultos. En la actualidad existe un creciente cuerpo de profesionales internacionales que abogan por que los adolescentes con cáncer necesitan ser atendidos por profesionales que son expertos en su enfermedad junto a profesionales que son expertos en este grupo de edad.

Es importante que toda la experiencia con el cáncer, los jóvenes tengan la oportunidad de seguir viviendo una vida tan normal como sea posible, para efectuar las tareas de desarrollo, y participar en los muchos hitos que se producen durante esta etapa de la vida. Si los equipos de tratamiento pueden mostrar más flexibilidad y acercamiento a las posturas y necesidades del adolescente en el tratamiento, los momentos de consulta y los procedimientos, se puede recorrer un largo camino en favorecer la adherencia y la cementación de una relación de confianza con el paciente adolescente.

\section{REFERENCIAS BIBLIOGRÁFICAS}

1. Petersen AC, Kennedy RE, Sullivan P. Coping with adolescence. In Colten ME, Gore S, editors. Adolescent stress: Causes and consequences. Hawthorne, NY: Aldine de Gruyter, 1991.p.93-110.

2. Buitelaar JK. Adolescence as a turning point: for better and worse. Eur Child Adolesc Psychiatry 2012;21:357-9. Doi: 10.1007/s00787-012-0296-3.

3. Gavaghan MP, Roach JE. Ego identity development of adolescents with cancer. J Pediatr Psychol 1987;12:203-13. Doi: 10.1093/jpepsy/12.2.203

4. Havinghurst RJ. Developmental tasks and education. New York: David McKay, 1992

5. Abrams AN, Hazen EP, Penson RT. Psychosocial issues in adolescents with cancer. Cancer Treat Rev 2007;33:622-30. Doi:10.1016/j.ctrv.2006.12.006

6. Barr RD. The adolescent with cancer. Eur J Cancer 2001;37:1523-7. Doi:10.1016/ S0959-8049(01)00161-7

7. Desandes E. Survival from adolescent cancer. Cancer Treat Rev 2007;33:609-15. Doi:10.1016/j.ctrv.2006.12.007

8. Lewis IJ, Cancer in adolescence. Br Med Bull 1996;52:887-97.

9. Seitz DC, Besier T, Goldbeck L. Psychosocial interventions for adolescent cancer patients: A systematic review of the literature. Psychooncology 2009;18:683-90. Doi: 10.1002/pon.1473.

10. Offer D, Ostrov E, Howard KI. Adolescent: What is normal? Am J Dis Child 1989;143:731-6.

11. Jörngarden A, Mattsson E, von Essen L. Health-related quality of life, anxiety and depression among adolescents and young adults with cancer: a prospective longitudinal study. Eur J Cancer 2007;43:1952-8. Doi:10.1016/j.ejca.2007.05.031

12. Mattsson E, El-Khouri B, Ljungman G, von Essen, L. Empirically derived psychosocial states among adolescents diagnosed with cancer during the acute and extended pha- 
se of survival. Ann Oncol 2009;20:1722-

7. Doi: 10.1093/annonc/mdp066.

13. Collins JJ, Byrnes ME, Dunkel IJ, Lapin J, Nadel T, Thaler HT, et al. The measurement of symptoms in children with cancer. J Pain Symptom Manage 2000; 19:363-77. Doi:10.1016/S0885-3924(01)00375-X

14. Stiller CA, Desandes E, Danon SE, Izarzugaza I, Ratiu A, Vassileva-Valerianova Z. Cancer incidence and survival in European adolescents (1978-1997). Report from the Automated Childhood Cancer Information System Project. Eur J Cancer 2006;42: 2006-18. Doi:10.1016/j.ejca.2006.06.002

15. Spinetta JJ, Masera G, Jankovic M, Oppenheim D, Martins AG, Ben Arush MW, et al. Valid informed consent and participative decision-making in children with cancer and their parents: A report of the SIOP Working Committee on psychosocial issues in pediatric oncology. Med Pediatr Oncol 2003;40:244-6. Doi:10.1002/mpo.10262

16. Ferrari A, Bleyer A. Participation of adolescents with cancer in clinical trials. Cancer Treat Rev 2007;33:603-8. Doi:10.1016/j. ctrv.2006.11.005

17. Andersen BL, Cacioppo JT. Delay in seeking a cancer diagnosis: Delay stages and psychophysiological comparison processes. Br J Soc of Psychol 1995;34:33-52. Doi:10.1111/j.2044-8309.1995.tb01047.x

18. Kyungas HA, Kroll T, Duffy ME. Compliance in adolescents with chronic diseases: A review. J Adolesc Health 2000;26:379-88.

19. Selby P, Bailey C. Cancer and the adolescent. London: BMJ Publishing Group, 1996.

20. Kazak AE, Simms S, Rourke MT. Family systems practice in pediatric psychology. J Pediatric Psychol 2002;27:133-43. Doi:10.1093/jpepsy/27.2.133

21. Whyte F, Smith L. A literature review of adolescence and cancer. Eur J Cancer Care 1997;6:137-46. 10.1046/j.13652354.1997.00022.x

22. Wu E, Robison LL, Jenney ME, Rockwood $\mathrm{TH}$, Feusner J, Friedman D, et al. As- sessment of health related quality of life of adolescent cancer patients using the Minneapolis-Manchester Quality of Life Adolescent Questionnaire. Pediatric Blood Cancer 2007;48:678-86. Doi: 10.1002/ pbc. 20874

23. Koochar GP, Omally JE, Grogan JL, Foster DJ. Psychological adjustment among paediatric cancer survivors. J Child Psychol Psychiatry 1980;21:163-73.

24. Kashani J, Hakami N. Depression in children and adolescents with malignancy. Can J Psychiatry 1982;27:474-7.

25. Allen R, Newman SP, Souhami RL. Anxiety and depression in adolescent cancer: Findings in patients and parents at the time of diagnosis. Eur J Cancer 1997;33:1250-5. Doi:10.1016/S0959-8049(97)00176-7

26. Patenaude AF, Kupst MJ. Psychosocial functioning in pediatric cancer. J Pediatr Psychol 2005;30:9-27. Doi: 10.1093/jpepsy/jsi012

27. Seitz DCM, Besier T, Debatin KM, Grabow D, Dieluweit U, Hinz A, et al. Posttraumatic stress, depression and anxiety among adult long-term survivors of cancer in adolescence. Eur J Cancer 2010;46:1596-606. Doi: 10.1016/j.ejca.2010.03.001.

28. Evan EE, Kaufman M, Cook AB, Zeltzer LK. Sexual health and self-esteem in adolescents and young adults with cancer. Cancer 2006;107(7 Suppl):1672-9.

29. Pao M, Ballard ED, Rosenstein DL, Wiener L, Wayne AS. Psychotropic medication use in pediatric patients with cancer. Arch Pediatr Adolesc Med 2006;160:818-22. Doi:10.1001/archpedi.160.8.818.

30. Kersun LS, Kazak AE. Prescribing practices of selective serotoninre-uptake inhibitors (SSRIs) among pediatric oncologists: A single institution study. Pediatr Blood Cancer 2006;47:339-42. Doi:10.1002/pbc.20484

31. Bleyer WA. Cancer in the older adolescents and young adults: epidemiology, diagnosis, treatment, survival, and importance of clinical trials. Med Pediatr Oncol 2002;38:1-10. Doi:10.1002/mpo.1257 
32. Von Essen L, Enskär K, Kreuger A, Larsson B, Sjödén PO. Self-esteem, depression and anxiety among Swedish children and adolescents on and off cancer treatment. Acta Paediatr 2000;89: 229-36. Doi:10.1111/j.1651-2227.2000.tb01221.x

33. Kaplan SL, Busner J, Weinhold C, Lenon P. Depressive symptoms in children and adolescents with cancer: A longitudinal study. J Am Acad Child Adolesc Psychiatry 1987;26:782-7. Doi:10.1097/00004583198709000-00028

34. Sanger MS, Copeland DR, Davidson ER. Psychosocial adjustment among pediatric cancer patients: A multidimensional assessment. J Pediatr Psychol 1991;16:46374. Doi: 10.1093/jpepsy/16.4.463

35. Greenberg H, Kazak A, Meadows A. Psychological functioning in 8-16 yearold cancer survivors and their parents. J Paediatr 1989;114:488-93. Doi:10.1016/ S0022-3476(89)80581-5

36. Lungton, J. The nature of social support as experienced by women treated for breast cancer. J Adv Nurs 1997;25:1184-91. Doi: 10.1046/j.13652648.1997.19970251184.x

37. Butler RW, Mulhern RK. Neurocognitive interventions for children and adolescents surviving cancer. J Pediatr Psychol 2005;30:65-78. Doi: 10.1093/jpepsy/jsi017

38. Kyungas $H$, Mikkonen $R$, Nousiainen EM, Rytilahti $M$, Seppänen $P$, Vaattovaara $\mathrm{R}$, et al. Coping with the onset of cancer: Coping strategies and resources of young people with cancer. Eur J Cancer Care 2001;10:6-11. Doi: 10.1046/j.13652354.2001.00243.x

39. Engvall G, Mattsson E, von Essen L, Hedström M. Findings on how adolescents cope with cancer-a matter of methodology? Psychooncology 2011;20:1053-60. Doi: 10.1002/pon.1809.

40. Cantrell MA, Lupinacci P. A predictive model of hopefulness for adolescents. J Adolesc Health 2004;35:478-85. Doi:10.1016/j.jadohealth.2004.02.011
41. Hedström $M$, Kreuger $A$, Ljungman G, Nygren P, von Essen L. Accuracy of assessment of distress, anxiety, and depression by physicians and nurses in adolescents recently diagnosed with cancer. Pediatric Blood Cancer 2006;46:773-9. Doi: 10.1002/pbc.20693

42. Eccles JS, Midgley C, Wigfield A, Buchanan CM, Reuman D, Flanagan $C$, et al. Development during adolescence: The impact of stage-environment fit on young adolescents' experiences in schools and families. Am Psychol 1993;48:90-101. Doi:10.1037/0003-066X.48.2.90

43. Manne S, Miller D. Social support, social conflict, and adjustment among adolescents with cancer. J Pediatr Psychol 1998; 23:121-30. Doi: 10.1093/jpepsy/23.2.121

44. Pendley JS, Dahlquist LM, Dreyer Z. Body image and psychosocial adjustment in adolescent cancer survivors. J Pediatr Psychol 1997;22:29-43.

45. Felder-Puig $R$, Formann AK, Mildner A, Bretschneider W, Bucher B, Windhager $R$ et al. Quality of life and psychosocial adjustment of young patients after treatment of bone cancer. Cancer 1998;83:69-75. Doi: 10.1002/(SICI)10970142(19980701)83:1\%3C69::AIDCNCR10\%3E3.0.CO;2-A

46. Zebrack BJ, Foley S, Wittmann D, Leonard $M$. Sexual functioning in young adult survivors of childhood cancer. Psychooncology 2010;19:814-22. Doi: 10.1002/ pon.1641.

47. Morgan S, Davies S, Palmer S, Plaster M. Sex, drugs, and rock ' $\mathrm{n}$ ' roll: Caring for adolescents and young adults with cancer. J Clin Oncol 2010;28:4825-30. Doi: 10.1200/JCO.2009.22.5474.

48. Vannatta K, Gerhardt CA, Wells RJ, Noll RB. Intensity of CNS treatment for pediatric cancer: prediction of social outcomes in survivors. Pediatr Blood Cancer 2007;49:716-22. Doi: 10.1002/pbc.21062

49. Van Dijk EM, van Dulmen-den Broeder E, Kaspers GJL, van Dam EW, Braam 
$\mathrm{KI}$, Huisman J. Psychosexual functioning of childhood cancer survivors. Psychooncology 2008;17:506-11. Doi: 10.1002/ pon. 1274

50. Langeveld NE, Stam H, Grootenhuis MA, Last BF. Quality of life in young adult survivors of childhood cáncer. Support Care Cancer 2002;10:579-600.

51. Rauck AM, Green DM, Yasui Y, Mertens A, Robison LL. Marriage in the survivors of childhood cancer: A preliminary description from the childhood cancer survivor study. Med Pediatr Oncol 1999;33:60-63. Doi: 10.1002/ (SICI)1096-911X(199907)33:1<60::AIDMPO11>3.0.CO;2-H

52. Kokkonen J, Vainionpaa L, Winqvist S, Lanning M. Physical and psychosocial outcome for young adults with treated malignancy. Pediatr Hematol Oncol 1997;14:223-32.

53. Puukko LR, Sammallahti PR, Siimes MA, Aalberg VA. Childhood leukemia and body image: Interview reveals impairment not found with a questionnaire. J Clin Psychol 1997;53:133-7. Doi:10.1002/ (SICI)1097-4679(199702)53:2<133::AIDJCLP6>3.0.CO;2-U

54. Mackie E, Hill J, Kondryn H, McNally R. Adult psychosocial outcomes in long-term survivors of acute lymphoblastic leukaemia and Wihms' tumour: A controlled study. Lancet 2000;355:1310-4.

55. Dishion TJ, McMahon RJ. Parental monitoring and the prevention of child and adolescent problem behavior: A conceptual and empirical formulation. Clin Child Fam Psychol Rev 1998;1:61-75.

56. Madan-Swain A, Brown RT, Sexson SB, Baldwin K, Pais R, Ragab A. Adolescent cancer survivors: Psychosocial and familial adaptation. Psychosomatics 1994; 5:45-9. Doi:10.1016/S0033-3182(94)71739-4

57. Schover LR. Sexuality and fertility after cancer. Hematology 2005;1:523-527.

58. Aslam I, Fishel S, Moore H, Dowell K, Thornton S. Fertility preservation of boys undergoing anti-cancer therapy: A review of the existing situation and prospects for the future. Hum Reprod 2000;15:2154-9. Doi:10.1093/humrep/15.10.2154

59. Committee opinion no.607. Gynecologic concerns in children and adolescents with cancer. Obstet Gynecol 2014;124;403-8. Doi: 10.1097/01. AOG.0000452746.44206.18.

60. Quinn GP, Murphy D, Kanapp C, Stearsman DK, Bradley-Klug KL, Sawczyn K, Clayman ML. Who Decides? Decision making and fertility preservation in teens with cancer: A review of the literature. J Adolescent Health 2011;49:337-46. Doi: 10.1016/j.jadohealth.2011.01.005.

61. Chapple A, Salinas M, Ziebland S, McPherson A, MacFarlane A. Fertility issues: The perceptions and experiences of young men recently diagnosed and treated for cancer. J Adolesc Health 2007;40:69-75. Doi:10.1016/j.jadohealth.2006.07.010

62. Wallace WHB, Anderson RA, Irvine DS. Fertility preservation for young patients with cancer: Who is at risk and what can be offered. Lancet Oncol 2005;6:209-18. Doi: 10.1016/S1470-2045(05)70092-9

63. Kazak AE, Derosa BW, Schwartz LA, Hobbie W, Carlson C, Ittenbach RF, et al. Psychological outcomes and health beliefs in adolescent and young adult survivors of childhood cancer and controls. J Clin Oncol 2010;28:2002-7. Doi: 10.1200/ JCO.2009.25.9564.

64. Stam H, Grootenhuis MA, Caron HN, Last BF. Quality of life and current coping in young adult survivors of childhood cancer: Positive expectations about the further course of the disease were correlated with better quality of life. Psychooncology 2006;15:31-43. Doi:10.1002/pon.920

65. Van Dongen-Melman JE. Developing psychosocial aftercare for children surviving cancer and their families. Acta Oncol 2000;39:23-31.

66. Canada AL, Schover LR, Li Y. A pilot intervention to enhance psychosexual de- 
velopment in adolescents and young adults with cancer. Pediatr Blood Cancer 2007;49:824-8. Doi:10.1002/pbc.21130

67. Baider L, Kaplan De-Nour A. Group therapy with adolescent cancer patients. J Adolesc Health Care 1989;10:35-8.

68. Cartwright-Hatton S, Roberts C, Chitsabesan P, Fothergill C, Harrington R. Systematic review of the efficacy of cognitive behaviour therapies for childhood and adolescent anxiety disorders. Br J Clin Psychol 2004;43:421-36. Doi:10.1348/0144665042388928

69. Compton SN, March JS, Brent D, Albano AM 5th, Weersing R, Curry J. Cognitive-behavioral psychotherapy for anxiety and depressive disorders in children and adolescents: An evidence-based medicine review. J Am Acad Child Adolesc Psychiatry 2004;43:930-59.

70. Butler AC, Chapman JE, Forman EM, Beck AT. The empirical status of cognitive-behavioral therapy: A review of meta-analyses. Clin Psychol Rev 2006;26:17-31. Doi:10.1016/j.cpr.2005.07.003

71. Sansom-Daly UM, Peate M, Wakefield CE, Bryant RA, Cohn RJ. A Systematic Review of psychological interventions for adolescents and young adults living with chronic illness. Health Psychol 2011;31:380-93. Doi: 10.1037/a0025977.

72. Butow PN, Kirsten LT, Ussher JM, Wain GV, Sandoval M, Hobbs KM et al. What is the ideal support group? Views of Australian people with cancer and their carers. Psychooncology 2007;16:1039-45. Doi:10.1002/pon. 1172

73. Roberts CS, Piper L, Denny J, Cuddeback G. A support group intervention to facilitate young adults' adjustment to cancer. Health Soc Work 1997;22:133-41.
74. Santacroce SJ, Asmus K, Kadan-Lottick N, Grey M. Feasibility and preliminary outcomes from a pilot study of coping skills training for adolescent-young adult survivors of childhood cancer and their parents. J Pediatr Oncol Nurs 2010;7:1020. Doi: 10.1177/1043454209340325.

75. Schiffman JD, Csongradi E, Suzuki LK. Internet use among adolescent and young adults (AYA) with cancer. Pediatr Blood Cancer 2008;51:410-5. Doi: 10.1002/ pbc. 21616.

76. Rickwood DJ, Deane FP, Wilson CJ. When and how do young people seek professional help for mental health problems? Med J Aust 2007;187(7 Suppl):S35-S39.

77. Sansom-Daly UM, Wakefield CE, Bryant RA, Butow P, Sawyer S, Patterson P, et al. Online group-based cognitive-behavioural therapy for adolescents and young adults after cancer treatment: A multicenter randomised controlled trial of Recapture Life-AYA. BMC Cancer 2012;12:339. Doi: 10.1186/1471-2407-12-339.

78. Shama W, Lucchetta S. Psychosocial issues of the adolescent cancer patient and the development of the Teenage Outreach Program (TOP). J Psychosoc Oncol 2007;25:99-112. Doi: 10.1300/ J077v25n03_06.

79. Barrera M, Petingola S, Fleming C, Mayer J. Support and intervention groups for adolescents with cancer in two Ontario communities. Cancer 2006; 107(7 Suppl.):1680-5.

80. Seitz DC, Besier T, Goldbeck L. Psychosocial interventions for adolescent cancer patients: A systematic review of the literature. Psychooncology 2009;18:683-90. Doi: 10.1002/pon.1473 